\title{
Sufficiency for general hypergeometric transform associated with conic region
}

\author{
Waseem A. Khan ${ }^{1}$, Idrees A. Khan ${ }^{2}$, Moin Ahmad ${ }^{3}$ and K.S. Nisar ${ }^{4}$ \\ 1,2,3 Department of Mathematics, Faculty of Science, Integral University, Lucknow-226026 India \\ ${ }^{4}$ Department of Mathematics, Prince Sattam bin Abdulaziz University, Riyadh region 11991, Saudi Arabia
}

Received: 6 June 2018, Accepted: 27 July 2018

Published online: 212019.

Abstract: The main object of the present paper is to find conditions on $a, b, c$ and $\lambda$ such that the operator $H_{a, b, c}^{\lambda} f(z)$ maps certain sub classes of analytic functions in to some other classes of functions that have geometric properties related to certain conic regions.

Keywords: Univalent, Convex, Starlike, Gaussian hypergeometric functions, integral transformation.

\section{Introduction}

Let $A$ denote the class of functions $f(z)$ of the form

$$
f(z)=z+\sum_{n=2}^{\infty} a_{n} z^{n}
$$

which are analytic in the open $\operatorname{disc} U=\{z:|z|<1\}$ and $\mathrm{S}$ denotes the sub classes of the function of $A$, which are univalent in $U$. A function $f \in A$ is called star like of order $\alpha$, denotes $f \in S^{*}(\alpha)$, if

$$
R_{e}\left\{z \frac{f^{\prime}(z)}{f(z)}\right\}>\alpha, z \in U
$$

A function $f \in A$ is called convex of order $\alpha$, if and only if,

$$
R_{e}\left\{1+z \frac{f^{\prime \prime}(z)}{f^{\prime}(z)}\right\} \geq \alpha, z \in U
$$

The class of all convex functions of order $\alpha$, are denoted by $K(\alpha)$. The classes $K(\alpha)$ and $S^{*}(\alpha)$, where introduced and studied by Robertson [13]. For $\alpha=0$, the classes $S^{*}(\alpha)$ and $K(\alpha)$ reduced to the classes $S^{*}$ respectively. Let $S^{*}(\lambda>0)$ denotes the class of functions in $\mathrm{S}$ such that

$$
\left|z \frac{f^{\prime}(z)}{f(z)}-1\right|<\lambda
$$

* Corresponding author e-mail: waseem08_khan@ @rediffmail.com 
A sufficient condition for $f \in A$ of the form (1) to be $S_{1}^{*} \subset S^{*}$, the class of starlike functions in $U$, is given by $\sum_{n=2}^{\infty} n\left|a_{n}\right| \leq 1$, and is proved by many authors for example (see [6]). A particular extension of this, due to [16] is

$$
\sum_{n=2}^{\infty}(n+\lambda-1)\left|a_{n}\right| \leq 1 \Rightarrow f \in S_{\lambda}^{*}
$$

We further note that when $f(z)$ is of the form (2), the condition (2) is both necessary and sufficient for $f \in S_{\lambda}^{*}$.

Definition 1. [4] Let $f \in A, 0 \leq k<\infty$ and $0 \leq \alpha<1$. Then $f \in k-U C V(\alpha)$ if and only if

$$
R_{e}\left\{1+z \frac{f^{\prime \prime}(z)}{f^{\prime}(z)}\right\} \geq\left|z \frac{f^{\prime \prime}(z)}{f^{\prime}(z)}\right|+\alpha .
$$

This class generalizes various other classes which are worthy of mention here. The class $k-U C V(0)$ called the $\mathrm{k}$-Uniformly convex is to [8] and has a geometric characterization given in the following way.

Let $0 \leq k<\infty$, the function $f \in A$ is said to to be k-Uniformly convex in $U$ if $f$ is convex in $U$ and the image of every circular arc $\gamma$ contained in $U$, with center $\xi$ where $|\xi| \leq k$, in convex.

The class $U C V(0)=U C V$, [5] describes geometrically the domain of values of the expression $P(z)=\left\{1+z \frac{f^{\prime \prime}(z)}{f^{\prime}(z)}, z \in U\right\}$ as $f \in U C V$ if and only if $\mathrm{P}$ is the conic region

$$
\Omega=\left\{w \in C:(\operatorname{Im} w)^{2}<2 R_{e} w-1\right\}
$$

Using the Alexander transformation, we can obtain the class $k-S_{p}(0)$ in the following way if $f \in k-U C V(\alpha) \Leftrightarrow z f^{\prime} \in k-S_{p}(\alpha)$.

The classes $U C V$ and $S_{P}:\left(1-S_{p}(0)\right)$ are unified and studied using a certain fractional calculus operator in [16], we refer the reader to $[9,7,14]$ and references there in for some interesting results in these directions.

Definition 2. The Gaussian Hypergeometric function

$$
{ }_{2} F_{1}(a, b ; c ; z)=\sum_{n=0}^{\infty} \frac{(a)_{n}(b)_{n}}{(c)_{n}} \frac{z^{n}}{n !},|z|<1
$$

where $(a)_{0}=1,(a)_{n+1}=(a+n)(a)_{n}, n=0,1,2, \cdots$ has appeared in the literature in many situations and contributed to various including conformal mappings, quasi conformal theory, and continued fractions [3,4]. Here a,b,c are complex numbers and $\{C \neq 0,-1,-2,-3, \cdots\}$. In the case of $a=-k$, or $b=-k$, where $J=0,1,2, \cdots$, and $K \leq m$ in this case $F(a, b ; c ; z)$ becomes a polynomials of degree $K$, we refer to a hypergeometric polynomials. The hypergeometric functions satisfies numbers and we remark that the behavior of the hypergeometric functions $F(a, b ; c ; z)$ near $z=1$, is classified in to three case according as $R_{e}(c-b-a)$ is positive, zero or negative. The case $c=a+b$ is called zero balanced case and hypergeometric $R_{e}(c) \leq R_{e}(a+b)$ as the asymptotic behavior in two case $a+b=c$ and $a+b>c$ has been refined in [1] and $[12]$ respectively.

If $R_{e}(c-a-b)>0($ see [18]), then

$$
F(a, b ; c ; 1)=\frac{\Gamma(c-a-b) \Gamma(c)}{\Gamma(c-a) \Gamma(c-b)} .
$$


In this paper, we introduce the operator $H_{a, b ; c}^{\lambda} f(z)$ such that

$$
\begin{aligned}
H_{a, b ; c}^{\lambda} f(z) & =\lambda z F(a, b ; c ; z)+(1-\lambda) n z[F(a, b ; c ; z)] * f(z) \\
& =\lambda\left[z+\sum_{n=2}^{\infty} \frac{(a)_{n-1}(b)_{n-1}}{(c)_{n-1}(n-1) !} z^{n}\right]+(1-\lambda) n\left[z+\sum_{n=2}^{\infty} \frac{(a)_{n-1}(b)_{n-1}}{(c)_{n-1}(n-1) !} z^{n}\right] * f(z) \\
& =\left[z+\sum_{n=2}^{\infty} \frac{(a)_{n-1}(b)_{n-1}}{(c)_{n-1}(n-1) !} z^{n}\right]\{\lambda+n(1-\lambda)\} * f(z)
\end{aligned}
$$

where

$$
A_{n}=\frac{(a)_{n-1}(b)_{n-1}}{(c)_{n}-1(n-1) !}\{\lambda+n(1-\lambda)\} a_{n}
$$

\section{Main results and preliminary lemmas}

We state a few results obtained in the literature by various author which are useful in proving our results.

Let $\tau=e^{i \eta} \cos \eta$, where $-\frac{\pi}{2}<\eta<\frac{\pi}{2}$, then we have $P_{\gamma}^{\tau}(\beta)=P_{\gamma}(\beta)$, which can be written as the following simple analytic characterization:

$$
P_{\gamma}(\beta)=\left\{f \in A: R_{e}\left[e^{i \phi}\left((1-\gamma) \frac{f(z)}{z}+\gamma f^{\prime}(z)-\beta\right)\right]>0, \phi \in R, z \in U\right\}
$$

Throughout this paper by $P_{\gamma}(\beta)$, we mean $P_{\beta}^{\tau}$ with $\tau=e^{i \eta} \cos \eta$ where $-\frac{\pi}{2}<\eta<\frac{\pi}{2}$ we need the following sufficient condition on the sufficient of the class $k-U C V(\alpha)$.

Lemma 1. [4] A function $f \in A$ is in $k-U C V(\alpha)$ if it satisfied the condition

$$
\sum_{n=2}^{\infty} n[n(1+k)-(k+\alpha)]\left|a_{n}\right| \leq 1-\alpha
$$

It was also found that the condition (10) is necessary, if $f \in A$ is of the form

$$
f(z)=z-\sum_{n=2}^{\infty} a_{n} z^{n}, a_{n} \geq 0
$$

Further more, the condition

$$
\sum_{n=2}^{\infty}[n(1+k)-(k+\alpha)]\left|a_{n}\right| \leq 1-\alpha .
$$

Is sufficient for $f$ to be in $k-S_{p}(\alpha)$ and turns out to be also necessary if $f \in A$ is of the form (10).

Theorem 1. Let $f \in A$ be defined as in (1). Suppose that $a, b \in C /\{0\}, C>|a|+|b|$ are such that for $k \geq 0$, $\lambda \geq 0$, $0 \leq \alpha<1$

$$
\begin{aligned}
& \frac{\Gamma(c-|a|-|b|) \Gamma(c)}{\Gamma(c-|a|) \Gamma(c-|b|)}\left[\{(1+k)(2-\lambda)+(1-\lambda)(1-\alpha)\} \frac{|a b|}{(c-|a|-|b|-1)}\right. \\
& \left.+(1-\alpha)+(1+k)(1-\lambda) \frac{|a b|(|a|+1)(|b|+1)}{(c-|a|-|b|-2)(c-|a|-|b|-1)}\right] \leq(1-\alpha)\left\{1+\frac{\gamma}{2 \cos \eta(1-\beta)}\right\}
\end{aligned}
$$

then for $f \in p_{\gamma}(\beta), 0 \leq \alpha \leq 1$ and $0 \leq \beta \leq 1, H_{a, b, c}^{\lambda} f(z) \in k-U C V(\alpha)$. 
Proof. Let $f \in A$ be defined as in theorem (10). From a result given in [18], we have

$$
f(z)=z+\sum_{n=2}^{\infty} a_{n} z^{n} \in P_{\gamma}(\beta) \Rightarrow\left|a_{n}\right| \leq \frac{2|\tau|(1-\beta)}{1+\gamma(n-1)}
$$

Considering (10), we need to prove that if $f \in A$ satisfies (11), then

$$
\sum_{n=2}^{\infty} n\{n(1+k)-(k+\alpha)\}\left|A_{n}\right| \leq 1-\alpha,
$$

where

$$
\begin{gathered}
A_{n}=\frac{(a)_{n-1}(b)_{n-1}}{(c)_{n-1}(n-1) !}\{\lambda+n(1-\lambda)\} a_{n}, n \geq 2 . \\
\sum_{n=2}^{\infty} n\{n(1+k)-(k+\alpha)\} \frac{(|a|)_{n-1}(|b|)_{n-1}}{(c)_{n-1}(n-1) !}\{\lambda+n(1-\lambda)\} \frac{2 \cos \eta(1-\beta)}{1+\gamma(n-1)} \leq 1-\alpha .
\end{gathered}
$$

Since $1+\gamma(n-1) \geq \gamma n$ and $n \geq 2$

$$
\begin{aligned}
& \leq \sum_{n=2}^{\infty} n\{n(1+k)-(k+\alpha)\} \frac{(|a|)_{n-1}(|b|)_{n-1}}{(c)_{n-1}(n-1) !} \frac{\{\lambda+n(1-\lambda)\}}{\gamma n} \leq \frac{(1-\alpha)}{2 \cos \eta(1-\beta)} \\
& =\sum_{n=2}^{\infty}\{n(1+k)-(k+\alpha)\} \frac{(|a|)_{n-1}(|b|)_{n-1}}{(c)_{n-1}(n-1) !}\{\lambda+n(1-\lambda)\} \leq \frac{\gamma(1-\alpha)}{2 \cos \eta(1-\beta)} .
\end{aligned}
$$

Replacing $n$ by $(n+2)$,we get

$$
\sum_{n=0}^{\infty}\{(n+2)(1+k)-(k+\alpha)\} \frac{(|a|)_{n+1}(|b|)_{n+1}}{(c)_{n+1}(n+1) !}\{\lambda+(n+2)(1-\lambda)\} .
$$

Using

$$
\begin{aligned}
& \{(n+2)(1+k)-(k+\alpha)\}=\{(n+1)(1+k)+(1-\alpha)\} \\
& =\sum_{n=0}^{\infty}\{(n+1)(1+k)+(1-\alpha)\} \frac{(|a|)_{n+1}(|b|)_{n+1}}{(c)_{n+1}(n+1) !}\{\lambda+(n+2)(1-\lambda)\} \\
& =(1+k) \sum_{n=0}^{\infty}(n+1) \frac{(|a|)_{n+1}(|b|)_{n+1}}{(c)_{n+1}(n+1) !}\{\lambda+(n+2)(1-\lambda)\}+(1-\alpha) \sum_{n=0}^{\infty} \frac{(|a|)_{n+1}(|b|)_{n+1}}{(c)_{n+1}(n+1) !}\{\lambda+(n+2)(1-\lambda)\} \\
& =\lambda(1+k) \sum_{n=0}^{\infty} \frac{(|a|)_{n+1}(|b|)_{n+1}}{(c)_{n+1} n !}+(1-\lambda)(1+k) \sum_{n=0}^{\infty}(n+2) \frac{(|a|)_{n+1}(|b|)_{n+1}}{(c)_{n+1} n !} \\
& +\lambda(1-\alpha) \sum_{n=0}^{\infty} \frac{(|a|)_{n+1}(|b|)_{n+1}}{(c)_{n+1}(n+1) !}+(1-\lambda)(1-\alpha) \sum_{n=0}^{\infty}(n+2) \frac{(|a|)_{n+1}(|b|)_{n+1}}{(c)_{n+1}(n+1) !} \\
& =\lambda(1+k) \frac{|a b|}{c} \sum_{n=1}^{\infty} \frac{(|a|+1)_{n}(|b|+1)_{n}}{(c+1)_{n} n !}+(1-\lambda)(1+k) \sum_{n=0}^{\infty} \frac{(|a|)_{n+1}(|b|)_{n+1}}{(c)_{n+1}(n-1) !} \\
& +2(1-\lambda)(1+k) \frac{|a b|}{c} \sum_{n=1}^{\infty} \frac{(|a|+1)_{n}(|b|+1)_{n}}{(c+1)_{n} n !}+(1-\lambda)(1-\alpha) \frac{|a b|}{c} \sum_{n=1}^{\infty} \frac{(|a|+1)_{n}(|b|+1)_{n}}{(c+1)_{n}(n) !} \\
& +(1-\lambda)(1-\alpha) \sum_{n=0}^{\infty} \frac{(|a|)_{n+1}(|b|)_{n+1}}{(c)_{n+1}(n+1) !}+\lambda(1-\alpha) \sum_{n=1}^{\infty} \frac{(|a|)_{n}(|b|)_{n}}{(c)_{n} n !} \\
& =\{\lambda(1+k)+2(1-\lambda)(1+k)+(1-\lambda)(1-\alpha)\} \frac{|a b|}{c} \sum_{n=1}^{\infty} \frac{(|a|+1)_{n}(|b|+1)_{n}}{(c+1)_{n} n !}
\end{aligned}
$$




$$
\begin{aligned}
& +\left\{(1-\lambda)(1+k) \frac{(|a b|)(|a|+1)(|b|+1)}{(c)(c+1)}\right\} \sum_{n=2}^{\infty} \frac{(|a|+2)_{n-1}(|b|+2)_{n-1}}{(c+2)_{n-1} n-1 !} \\
& +\{\lambda(1-\alpha)+(1-\lambda)(1-\alpha)\} \sum_{n=1}^{\infty} \frac{(|a|)_{n}(|b|)_{n}}{(c)_{n} n !} \\
& =\{(1+k)(2-\lambda)+(1-\lambda)(1-\alpha)\}|a b| \frac{\Gamma c-|a|-|b|-1 \Gamma c}{\Gamma c-|a| \Gamma c-|b|} \\
& +\{(1-\lambda)(1+k)(|a b|)(|a|+1)(|b|+1)\} \\
& \times \frac{\Gamma c-|a|-|b|-2 \Gamma c}{\Gamma c-|a| \Gamma c-|b|}+(1-\alpha)\left\{\frac{\Gamma c-|a|-|b| \Gamma c}{\Gamma c-|a| \Gamma c-|b|}-1\right\} \\
& =\frac{\Gamma(c-|a|-|b|) \Gamma(c)}{\Gamma(c-|a|) \Gamma(c-|b|)}\left[\{(1+k)(2-\lambda)+(1-\lambda)(1-\alpha)\} \frac{|a b|}{(c-|a|-|b|-1)}\right. \\
& \left.+(1-\alpha)+(1+k)(1-\lambda) \frac{|a b|(|a|+1)(|b|+1)}{(c-|a|-|b|-2)(c-|a|-|b|-1)}\right] \leq(1-\alpha)\left\{1+\frac{\gamma}{2 \cos \eta(1-\beta)}\right\} .
\end{aligned}
$$

Remark. For $\lambda=1$ in theorem 2.1, the result reduces to the known result of Swaminathan [15].

Corollary 1. Let $f \in A$ be defined as in (1). Suppose that $a, b \in C \backslash\{0\}, C>|a|+|b|$ are such that for $k \geq 0,0 \leq \alpha<1$,

$$
\frac{\Gamma(c-|a|-|b|-1) \Gamma(c)}{\Gamma(c-|a|) \Gamma(c-|b|)}\{|a b|(1+k)+(1-\alpha)(c-|a|-|b|-1)\} \leq(1-\alpha)\left\{1+\frac{\gamma}{2 \cos \eta(1-\beta)}\right\},
$$

then for $f \in P_{\gamma}(\beta), 0 \leq \gamma \leq 1$ and $0 \leq \beta<1, H_{a, b, c}^{\lambda} f(z) \in k-U C V(\alpha)$.

Theorem 2. Let $a, b>0$ or $a \in C /\{0\}$ with $a=b$. Further, let $|a| \neq 0,|b| \neq 0$ and $0 \neq c \geq\{0, a+b-1\}$ be such that

$$
\begin{aligned}
\frac{\Gamma(c-|a|-|b|-1) \Gamma(c)}{\Gamma(c-|a|) \Gamma(c-|b|)} \times & {\left[\{1+(\mu-1)(1-\lambda)\}(c-|a|-|b|-1)+(1-\lambda)(|a b|)+\frac{\lambda(\mu-1)}{(|a|-1)(|b|-1)}\right] } \\
& \leq\left(\mu\left(1+\frac{\gamma}{2 \cos \eta(1-\beta)}\right)+\frac{\lambda(\mu-1)(c-1)}{(|a|-1)(|b|-1)}\right) .
\end{aligned}
$$

Assume that $f \in A$ is defined in (1). Then for $f \in P_{\gamma}(\beta), 0 \leq \gamma<1$ and $0 \leq \beta<1$ and $\lambda \geq 0, \mu \geq 0, H_{a, b, c}^{\lambda} f(z) \in S_{\lambda}^{*}$.

Proof. Let $f(z)$ be of the form (1). In view of (2.2), it suffices to prove that

$$
\sum_{n=2}^{\infty}(n+\mu-1)\left|A_{n}\right| \leq \mu
$$

where

$$
A_{n}=\frac{(a)_{n-1}(b)_{n-1}}{(c)_{n-1}(n-1) !}\{\lambda+n(1-\lambda)\} a_{n}, n \geq 2
$$


Since $f \in P_{\gamma}(\beta)$, using $(2.2)$ and $1+\gamma(n-1) \geq \gamma n$ we need only to show that

$$
\begin{aligned}
& T=\sum_{n=2}^{\infty}(n+\mu-1) \frac{(|a|)_{n-1}(|b|)_{n-1}}{(c)_{n-1}(n-1) !}\{\lambda+n(1-\lambda)\} \frac{2 \cos \eta(1-\beta)}{1+\gamma(n-1)} \leq \mu \\
& \leq \sum_{n=2}^{\infty}(n+\mu-1) \frac{(|a|)_{n-1}(|b|)_{n-1}}{(c)_{n-1}(n-1) !}\{\lambda+n(1-\lambda)\} \frac{1}{\gamma n} \leq \frac{\mu}{2 \cos \eta(1-\beta)} \\
& =\sum_{n=2}^{\infty}(n+\mu-1) \frac{(|a|)_{n-1}(|b|)_{n-1}}{(c)_{n-1}(n-1) !}\{\lambda+n(1-\lambda)\} \frac{1}{n} \leq \frac{\gamma \mu}{2 \cos \eta(1-\beta)} \\
& =\sum_{n=2}^{\infty} \frac{(|a|)_{n-1}(|b|)_{n-1}}{(c)_{n-1}(n-1) !}\{\lambda+n(1-\lambda)\}+(\mu-1) \sum_{n=2}^{\infty} \frac{(|a|)_{n-1}(|b|)_{n-1}}{(c)_{n-1} n !}\{\lambda+n(1-\lambda)\} \\
& =\lambda \sum_{n=2}^{\infty} \frac{(|a|)_{n-1}(|b|)_{n-1}}{(c)_{n-1}(n-1) !}+(1-\lambda) \sum_{n=2}^{\infty} \frac{(n-1+1)(|a|)_{n-1}(|b|)_{n-1}}{(c)_{n-1}(n-1) !} \\
& +(\mu-1) \lambda \sum_{n=2}^{\infty} \frac{(|a|)_{n-1}(|b|)_{n-1}}{(c)_{n-1} n !}+(1-\lambda)(\mu-1) \sum_{n=2}^{\infty} \frac{(|a|)_{n-1}(|b|)_{n-1}}{(c)_{n-1}(n) !} \\
& =\lambda \sum_{n=1}^{\infty} \frac{(|a|)_{n}(|b|)_{n}}{(c)_{n} n !}+(1-\lambda) \sum_{n=2}^{\infty} \frac{(|a|)_{n-1}(|b|)_{n-1}}{(c)_{n-1}(n-2) !}+(1-\lambda) \sum_{n=1}^{\infty} \frac{(|a|)_{n}(|b|)_{n}}{(c)_{n} n !} \\
& +(\mu-1) \lambda \frac{(c-1)}{(|a|-1)(|b|-1)} \sum_{n=2}^{\infty} \frac{(|a|-1)_{n}(|b|-1)_{n}}{(c-1)_{n} n !}+(1-\lambda)(\mu-1) \sum_{n=1}^{\infty} \frac{(|a|)_{n}(|b|)_{n}}{(c)_{n} n !} \\
& =\{\lambda+(1-\lambda)(\mu-1)+1-\lambda\} \sum_{n=1}^{\infty} \frac{(|a|)_{n}(|b|)_{n}}{(c)_{n} n !} \\
& +(1-\lambda) \frac{|a b|}{c} \sum_{n=1}^{\infty} \frac{(|a|+1)_{n-1}(|b|+1)_{n-1}}{(c+1)_{n-1}(n-1) !}+(\mu-1) \lambda \frac{(c-1)}{(|a|-1)(|b|-1)} \sum_{n=2}^{\infty} \frac{(|a|-1)_{n}(|b|-1)_{n}}{(c-1)_{n} n !} \\
& =\{1+(\mu-1)(1-\lambda)\}\left\{\frac{\Gamma(c-|a|-|b|) \Gamma(c)}{\Gamma(c-|a|) \Gamma(c-|b|)}-1\right\} \\
& +(|a b|)(1-\lambda) \frac{\Gamma(c-|a|-|b|-1) \Gamma(c)}{\Gamma(c-|a|) \Gamma(c-|b|)}+(\mu-1) \lambda \frac{(c-1)}{(|a|-1)(|b|-1)} \\
& \times\left\{\frac{\Gamma(c-|a|-|b|-1) \Gamma(c-1)}{\Gamma(c-|a|) \Gamma(c-|b|)}-1-\frac{(|a|-1)(|b|-1)}{c-1}\right\} \\
& =\frac{\Gamma(c-|a|-|b|-1) \Gamma(c)}{\Gamma(c-|a|) \Gamma(c-|b|)} \times\left[\{1+(\mu-1)(1-\lambda)\}(c-|a|-|b|-1)+(1-\lambda)(|a b|)+\frac{\lambda(\mu-1)}{(|a|-1)(|b|-1)}\right] \\
& \leq\left(\mu\left(1+\frac{\gamma}{2 \cos \eta(1-\beta)}\right)+\frac{\lambda(\mu-1)(c-1)}{(|a|-1)(|b|-1)}\right) \text {. }
\end{aligned}
$$

Remark. Setting $\lambda=1$ in theorem (11), the result reduces to the known result of Swaminathan [15].

Corollary 2. Let $a, b>0$ or $a \in C \backslash\{0\}$ with $a=b$. Further, let $|a| \neq 0,|b| \neq 0$ and $0 \neq c \geq\{0, a+b-1\}$ be such that

$$
\begin{aligned}
& \frac{\Gamma(c-|a|-|b|-1) \Gamma(c)}{\Gamma(c-|a|) \Gamma(c-|b|)} \times\left\{1+(\mu-1)(c-|a|-|b|-1)+(1-\lambda)(|a b|)+\frac{\lambda(\mu-1)}{(|a|-1)(|b|-1)}\right\} \\
& \leq\left\{\mu\left(1+\frac{\gamma}{2 \cos \eta(1-\beta)}\right)+\frac{\lambda(\mu-1)(c-1)}{(|a|-1)(|b|-1)}\right\} .
\end{aligned}
$$

Suppose that $f \in A$ is defined in (1). Then for $F \in P_{\gamma}(\beta), 0 \leq \gamma<1,0 \leq \beta<1$ and $\lambda \geq 0, \mu \geq 0, H_{a, b, c}^{\lambda}(f)(z) \in S_{\lambda}^{*}$. 
Theorem 3. Let $a, b>0$ and $0 \neq c \geq\{0, a+b+2\}$. If for $k \geq 0,0 \leq \alpha<1$

$$
\begin{aligned}
& \frac{\Gamma(c-a-b) \Gamma c}{\Gamma c-a \Gamma c-b}\left[(1-\alpha)+\left\{(1+k)(7-4 \lambda)-(k+\alpha)(3-2 \lambda) \frac{a b}{(c-a-b-1)}\right\}\right. \\
& \left.+\{\lambda(1+k)-(k+\alpha)(1-\lambda)+6(1+k)(1-\lambda)\} \times \frac{(a)_{2}(b)_{2}}{(c-a-b-2)_{2}}+(1+k)(1-\lambda) \frac{(a)_{3}(b)_{3}}{(c-a-b-3)_{3}}\right] \leq 2(1-\alpha) .
\end{aligned}
$$

Then $H_{a, b, c}^{\lambda} f(z)$ maps $f(z) \in S$ if the form (1) in yo $k-S_{p}(\alpha)$.

Proof. Using $\left|a_{n}\right| \leq n$ for $f(z) \in S$ and of the form (1). It is enough to show that

$$
\begin{aligned}
& T=\sum_{n=2}^{\infty}\{n(1+k)-(k+\alpha)\} \frac{(a)_{n-1}(b)_{n-1}}{(c)_{n-1}(n-1) !}\{\lambda+n(1-\lambda)\}\left|a_{n}\right| \leq 1-\alpha \\
& \leq \sum_{n=2}^{\infty}\left\{n^{2}(1+k)-(k+\alpha) n\right\} \frac{(a)_{n-1}(b)_{n-1}}{(c)_{n-1}(n-1) !}\{\lambda+n(1-\lambda)\} \\
& =\sum_{n=2}^{\infty} n^{2}(1+k) \frac{(a)_{n-1}(b)_{n-1}}{(c)_{n-1}(n-1) !}\{\lambda+n(1-\lambda)\}-(k+\alpha) \frac{n(a)_{n-1}(b)_{n-1}}{(c)_{n-1}(n-1) !}\{\lambda+n(1-\lambda)\} \\
& =\lambda(1+k) \sum_{n=2}^{\infty} n^{2} \frac{(a)_{n-1}(b)_{n-1}}{(c)_{n-1}(n-1) !}+(1+k)(1-\lambda) \sum_{n=2}^{\infty} n^{3} \frac{(a)_{n-1}(b)_{n-1}}{(c)_{n-1}(n-1) !} \\
& -(\alpha+k) \lambda \sum_{n=2}^{\infty} n \frac{(a)_{n-1}(b)_{n-1}}{(c)_{n-1}(n-1) !}-(\alpha+k)(1-\lambda) \sum_{n=2}^{\infty} n^{2} \frac{(a)_{n-1}(b)_{n-1}}{(c)_{n-1}(n-1) !} \\
& =\lambda(1+k) \sum_{n=1}^{\infty}(n+1)^{2} \frac{(a)_{n}(b)_{n}}{(c)_{n} n !}+(1+k)(1-\lambda) \sum_{n=1}^{\infty}(n+1)^{3} \frac{(a)_{n}(b)_{n}}{(c)_{n} n !} \\
& -(\alpha+k) \lambda \sum_{n=2}^{\infty}(n+1) \frac{(a)_{n}(b)_{n}}{(c)_{n} n !}-(\alpha+k)(1-\lambda) \sum_{n=1}^{\infty}(n+1)^{2} \frac{(a)_{n}(b)_{n}}{(c)_{n} n !} \\
& =\{\lambda(1+k)-(k+\alpha)(1-\lambda)\} \sum_{n=1}^{\infty}\{n(n-1)+3 n+1\} \frac{(a)_{n}(b)_{n}}{(c)_{n} n !} \\
& +(1+k)(1-\lambda) \sum_{n=1}^{\infty}\{n(n-1)(n-2)+6 n(n-1)+7 n+1\} \frac{(a)_{n}(b)_{n}}{(c)_{n} n !} \\
& -(\alpha+k) \lambda \sum_{n=1}^{\infty} \frac{(a)_{n}(b)_{n}}{(c)_{n}(n-1) !}-(\alpha+k) \lambda \sum_{n=1}^{\infty} \frac{(a)_{n}(b)_{n}}{(c)_{n} n !} \\
& =\{\lambda(1+k)-(k+\alpha)(1-\lambda)\}\left\{\sum_{n=1}^{\infty} \frac{(a)_{n}(b)_{n}}{(c)_{n}(n-2) !}+3 \sum_{n=1}^{\infty} \frac{(a)_{n}(b)_{n}}{(c)_{n}(n-1) !}+\sum_{n=1}^{\infty} \frac{(a)_{n}(b)_{n}}{(c)_{n} n !}\right\} \\
& +(1+k)(1-\lambda)\left\{\sum_{n=1}^{\infty} \frac{(a)_{n}(b)_{n}}{(c)_{n}(n-3) !}+6 \sum_{n=1}^{\infty} \frac{(a)_{n}(b)_{n}}{(c)_{n}(n-2) !}+7 \sum_{n=1}^{\infty} \frac{(a)_{n}(b)_{n}}{(c)_{n}(n-1) !}+\sum_{n=1}^{\infty} \frac{(a)_{n}(b)_{n}}{(c)_{n} n !}\right\} \\
& -(\alpha+k) \lambda \sum_{n=1}^{\infty} \frac{(a)_{n}(b)_{n}}{(c)_{n}(n-1) !}-(\alpha+k) \lambda \sum_{n=1}^{\infty} \frac{(a)_{n}(b)_{n}}{(c)_{n} n !} \\
& =(1-\lambda)(1+k) \sum_{n=1}^{\infty} \frac{(a)_{n}(b)_{n}}{(c)_{n}(n-3) !}+\{\lambda(1+k)-(k+\alpha)(1-\lambda)+6(1+k)(1-\lambda)\} \sum_{n=1}^{\infty} \frac{(a)_{n}(b)_{n}}{(c)_{n}(n-2) !} \\
& +\{3 \lambda(1+k)-3(k+\alpha)(1-\lambda)+7(1+k)(1-\lambda)-\lambda(k+\alpha)\} \sum_{n=1}^{\infty} \frac{(a)_{n}(b)_{n}}{(c)_{n}(n-1) !} \\
& +\{(1+k) \lambda-(k+\alpha)(1-\lambda)+(1+k)(1-\lambda)-(k+\alpha) \lambda\} \sum_{n=1}^{\infty} \frac{(a)_{n}(b)_{n}}{(c)_{n} n !}
\end{aligned}
$$




$$
\begin{aligned}
& =(1+k)(1-\lambda)_{3}(a)_{3}(b)_{3} \frac{\Gamma(c-a-b-3) \Gamma(c)}{\Gamma(c-a) \Gamma(c-b)}+\{\lambda(1+k)-(k+\alpha)(1-\lambda)+6(1+k)(1-\lambda)\} \\
& \times(a)_{2}(b)_{2} \frac{\Gamma(c-a-b-2) \Gamma c}{\Gamma c-a \Gamma c-b}+\{(1+k)(7-4 \lambda)-(k+\alpha)(3-2 \lambda)\} \\
& \times(a b) \frac{\Gamma(c-a-b-3) \Gamma c}{\Gamma c-a \Gamma c-b}+(1-\alpha)\left\{\frac{\Gamma(c-a-b) \Gamma c}{\Gamma c-a \Gamma c-b}-1\right\} \\
& =\frac{\Gamma(c-a-b) \Gamma c}{\Gamma c-a \Gamma c-b}\left[(1-\alpha)+\left\{(1+k)(7-4 \lambda)-(k+\alpha)(3-2 \lambda) \frac{a b}{(c-a-b-1)}\right\}\right. \\
& +\{\lambda(1+k)-(k+\alpha)(1-\lambda)+6(1+k)(1-\lambda)\} \\
& \left.\times \frac{(a)_{2}(b)_{2}}{(c-a-b-2)_{2}}+(1+k)(1-\lambda) \frac{(a)_{3}(b)_{3}}{(c-a-b-3)_{3}}\right] \leq 2(1-\alpha) .
\end{aligned}
$$

Remark. For $\lambda=1$ in theorem (12), the result reduces to the known result of Swaminathan [15].

Corollary 3. Let $a, b>0$ and $0 \neq c \geq\{0, a+b+2\}$. If for $k \geq 0,0 \leq \alpha<1$,

$$
\frac{\Gamma(c-a-b) \Gamma(c)}{\Gamma(c-a) \Gamma(c-b)}\left[(1-\alpha)(k+\alpha)(3-2 k-\alpha) \times \frac{a b}{(c-a-b-1)}+(1+k) \frac{(a)_{2}(b)_{2}}{(c-a-b-2)_{2}}\right] \leq 2(1-\alpha) .
$$

Then $H_{a, b, c}^{\lambda} f(z)$ maps $f(z) \in S$ of the form (1) in to $k-S_{p}(\alpha)$.

\section{Concluding remarks}

In this paper, we introduce the operator $H_{a, b, c}^{\lambda} f(z)$ and find the conditions $a, b, c$ and $\lambda$. The operator $H_{a, b, c}^{\lambda} f(z)$ maps certain subclass of analytic functions into some other classes of functions. By mean of Theorem 1, we have derived some (presumably) new operator as its special cases. It is noticed that, in a similar manner we can obtain various other useful operator with the help of Theorem 2. Also, it is remarked that if we replace the operator (6) (which is used to establish the main results) by any other operator, then we get a number of new interesting results.

\section{Competing interests}

The authors declare that they have no competing interests.

\section{Authors' contributions}

All authors have contributed to all parts of the article. All authors read and approved the final manuscript.

\section{References}

[1] Anderson, G. D, Baranard, R. W, Richards, K. C, Vamanamurthy, M. K and Vuorinen, M, Inequalities for zero- balanced Hypergeometric functions, Trans. Amer. Math. Soc. 347 (1995), 1713-1723.

[2] Anderson, G. D, Vamanamurty, M. K and Vuorinen M, Conformal invariants, inequalities, and quasi conformal maps , John-Wiley and Sons , 1997.

[3] Balasubramanian, Ponnusamy, R. S, Vuorinen, M, on Hypergeometric functions and functions spaces, J. Comput. Appl. Math. 139 (2002), 299-322. 
[4] Bharati, R, Parvatham, R, Swaminothan, on sub classes of uniformly convex functions and corresponding class of Star like functions, Tamkang J. Math. 28 (1997), 17-32.

[5] Goodman, A. W, on uniformly convex functions ,Ann. Polon. Math. 56 (1991), 87-92.

[6] Goodman, A. W, Univalent functions an non analytic curves, Proc. Amer. Math. Soc. 8 (1957), 598-601.

[7] Kanas, S, Wisniowaska, A, Conic regions and K-Starlike functions, Rev. Roumain Math. Pures Appl. 45 (2000), 647-657.

[8] Kanas, S, Wisniowaska, A, Conic regions and K- Uniformly convexity, J. Comput. Appl. Math. 105 (1999), 327-336.

[9] Kanas, S, Srivastava, H. M, Linear operator associated with K-Uniformly convex functions, integral transform Spec. Funct. 9 (2000), 121-132.

[10] Nehari, Z, Conformal Mapping, MC.Graw-Hill, New York, 1952.

[11] Ponnusamy, S, Ronning, F, Star likeness properties for convolutions involving Hypergeometric series, Ann. Univ. Mariae Curie. Sklodowaska L.II.1 (16)(1998), 141-155.

[12] Ponnusamy, S and Vuorinen, M, Asymptotic expansions and in equalities for Hypergeometric function, Mathematika, 44 (1997), 278-301.

[13] Robertson, M. S, On the theory of univalent functions, Ann. Math. 37 (1936), 374-408.

[14] Ronning, F, Uniformly Convex functions and corresponding class of starlike functions, Proc. Amer. Math. Soc. 18 (1993), $189-196$.

[15] Swaminathan, A, Certain sufficiency conditions on Gaussian Hypergeometric functions , J. Inequl. Pure Appl. Math. 5(4) (2004), 1-10. Article 83 (electronics)

[16] Srivastava, H. M, Mishra, A. k, Applications of fractional calculus to parabolic starlike and uniformly convex functions, Compute. Math. Appl. 39 (3-4)(2009), 57-69.

[17] Silverman, H, Univalent functions with negative coefficients, Proc. Amer. Math. Soc.51 (1975), 109-116.

[18] Temme, N. M, Special functions. An introduction to the classical functions of Mathematical physics, New York, Wiley, 1996. 